\title{
Enhanced Surveillance in a Digital Health Landscape: The Role of the Near- Real Time Right to Care Knowledge Centre within South Africa's APACE HIV Program
}

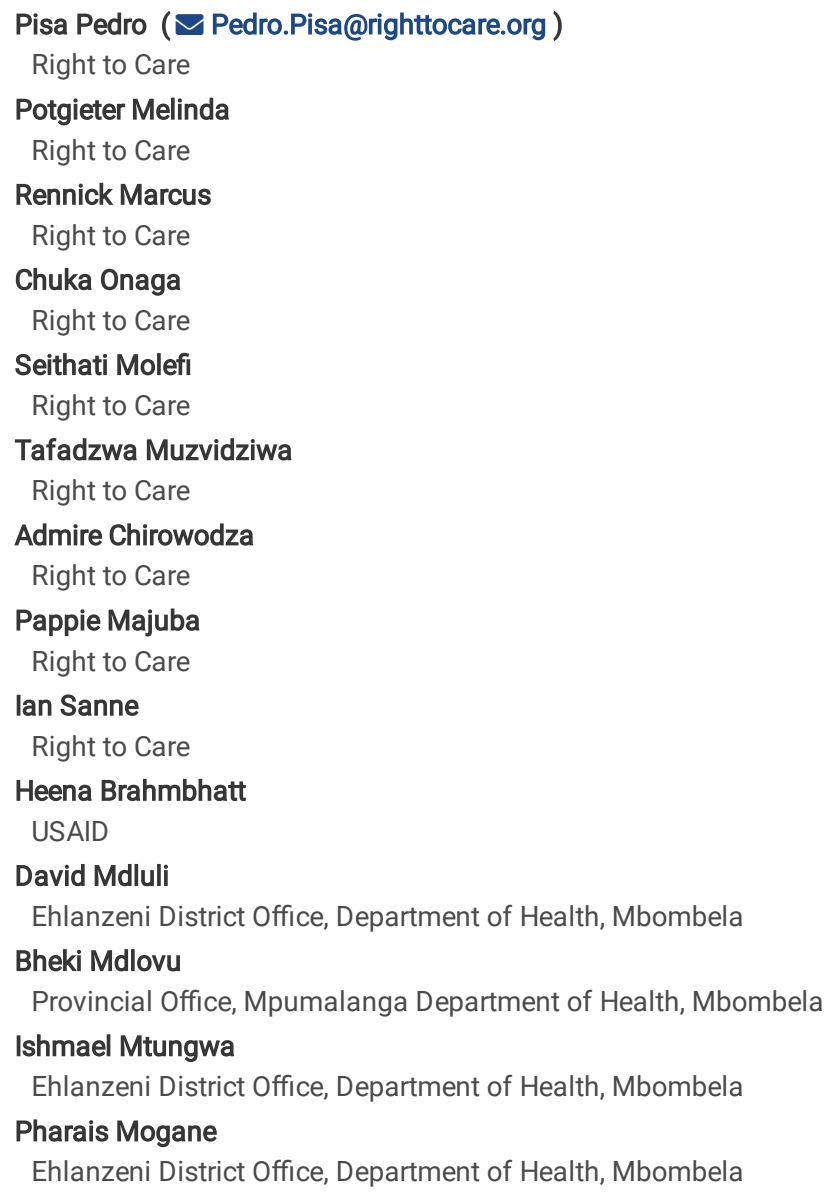




\section{Abstract}

Introduction: Right to Care (RTC), through support from the United States Agency for International Development (USAID) implements innovations that improve data accessibility and utilization for improved program performance and patient outcomes for large scale HIV care and treatment programs. Frequent and accessible data, coupled with changes to business practices to use that data, allow for targeted and timely HIV program interventions that impact patient outcomes in Ehlanzeni district, Mpumalanga, South Africa.

Methods: In South Africa, within the USAID Accelerating Program Achievements to Control the Epidemic (APACE) program, the Knowledge Centre (KC) - an interoperable automated data warehouse and visualization near real-time solution - allow for rapid daily assessment of over- and under-performance crosssectionally. The authors established the impact of the KC intervention for 29 USAID-selected Siyenza facilities, before the KC intervention (Dec 2018 to Feb 2019) compared to HIV outcomes during the KC intervention (March 2019 to May 2019), stratified by facility classification as included or not included in the Siyenza program using both non-parametric and parametric methodologies.

Results: Average facility ART initiations increased by $16 \%$ from a monthly average of 54 new initiations pre-Siyenza to 62 new ART initiations during Siyenza; retention increased to a net retention in care ratio of 1.15 indicating that patients labelled as lost to care were brought back. An independent-samples t-test indicated that the net retention in care scores were significantly higher for Siyenza facilities $(M=.379, S D=.808)$ compared to non-Siyenza facilities ( $M=-.061$, $S D=1.016), t(208)=2.224, p<0.027, d=.445$ during the Siyenza period. A significant difference was noted between the means with a $62.3 \%$ chance that the Siyenza facilities have a higher observed mean than the non-Siyenza facilities during the intervention period. Results indicate that Siyenza facilities maintain cohort growth, while non-Siyenza facilities continued to lose patients.

Conclusion: The accessibility and utilization of near to real-time interoperable data within HIV programs allowed for rapid responses to program performance, needs and improved patient outcomes. Daily data review meetings facilitated precision programming and contributed to the behavior change necessary to institute routine data use for decision making and evaluations within programs.

\section{Introduction}

Service delivery partners that support HIV care and treatment programs through the President's Emergency Plan for AIDS Relief (PEPFAR) track substantial data to demonstrate program performance across multiple indicators. These data are primarily collected retrospectively, aggregated, and used to passively report program achievements against funder-set targets as routine monitoring and evaluation activities (1). As countries and program work towards HIV epidemic control within their catchment areas (2), frequent data collection and utilization becomes more critical to identify lagging performance and prompt action. Retaining HIV infected patients in quality care, supports universal test and treat initiatives to eliminate new HIV infections (3) Regular review of data is critical to identify gaps in programs and indicates how to shift limited resources and interventions to improve patient outcomes proxied by performance against key indicator targets.

Bhattarai et al. (4) identified an urgent need for real-time data in improving processes and ultimately outcomes in healthcare and highlighted the need to reduce data lags to less than four weeks to re-duce patient losses and improve health outcomes.

Health Information Systems (HIS) can support facility-level quality improvement activities (5). Yet, even if these HIS are digitized, processing from facility to an aggregated data set takes valuable time. Further emphasis on perfect data quality for reporting purposes limits immediate action. Program quality improvement needs necessitate innovative solutions to make field data more accessible and actionable (6). More efficient data collection systems through automation allow limited resources to be directed to patient care rather than data collection (7). Effective data collection can, however, improve accessibility, relevance, accuracy, reliability, and coherence of the data. It also allows for the possibility to imbed data quality measures into the capturing, reporting and decision-making process.

Right to Care (RTC) operates as a care and service delivery partner under the United States Agency for International Development (USAID) Accelerating Program Achievements to Control the Epidemic (APACE) program in Ehlanzeni and Thabo Mofutsanyane districts in South Africa. We support 225 Department of Health facilities to provide quality HIV care and treatment to: (1) prevent new HIV infections and reduce HIV morbidity and mortality, (2) increase uptake of HIV testing, (3) increase the proportion of HIV-positive clients initiated on ART, and (4) increase the proportion of PLHIV who are virologically suppressed. USAID launched a special project known as Siyenza translated as "Let's do it", between 1st of March to the 9th of May 2019 in a bid to improve overall APACE performance, with a primary objective to rapidly accelerate program performance. To maximize existing resources, USAID and implementing partners selected sites with the greatest potential to improve overall cohort achievement. Specifically, Siyenza inclusion criteria considered facilities with the highest difference between expected outcomes (targets) and current achievement for new initiations and total patients in care in USAID-defined priority districts. Twenty-nine Right to Care-supported priority facilities from both the Thabo Mofutsanyane and Ehlanzeni districts were selected for the Siyenza initiative. The Siyenza initiative required an innovative approach to collect and act on data from across all supported facilities focusing on treatment initiations and retention. In the two mentioned districts, 29 high volume facilities contribute to some \% needed for patients remaining on ART contribution along with other facilities in the country.

\section{Methods}

Implementation data from Dec 2018 to May 2019 aggregated at facility level did not require consent. To reach the ambitious cohort targets needed to control the epidemic, a data collection and analysis platform was used in daily data review meetings with program managers and field staff in each district. The RTC Knowledge Centre (RTC KC) was used to collect, manage, and analyze the data using existing staffing infrastructures. The RTC KC is a Microsoft Azure-based warehouse and reporting platform developed in partnership with Qode Health Solutions. This platform supports HIV program management with big data

Page 2/9 
analytics and disparate data integration, including point of care testing data from HIV counsellors, custom program indicator data, laboratory results, facility HIS integrations, and other applicable data sources.

This data populates aggregated analytics accessible through an RTC KC web-portal that can be visualized at various levels of aggregation (age and gender stratifications by facility, district, and province) and used for monitoring program performance against set targets for various indicator and delivery improvement, targeted interventions resulting in improved clinical outcomes.

We chose to use Automated Data Capture (ADC) facility templates to support daily data collection and review for the Siyenza project. A daily template capturing the number of people tested for HIV (and those testing positive), initiated in treatment, and the total number of active clients on treatment was emailed to existing program data management staff based at each facility. The results in this report will, however, focus on patients-initiated on treatment and retained in care. The identified staff member completed the template at the end of the day and returned the completed template via email to an automated email server. Each file was then automatically integrated into the data warehouse populating visualizations used during data review meetings the next morning (Fig. 1).

The analysis was used during program managers' daily data review meetings to identify programming gaps in the facilities using data and to provide information from the KC. As a result, our analysis is currently limited to PEPFAR indicators related to patients-initiated onto ART and retention. The net retention is then compared to the number of new initiations as the net retention in care, TX_NET_NEW ratio, (Eq. 1).

Equation 1. Net retention in care ratio

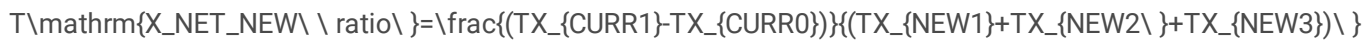

The net retention in care ratio or TX_NET_NEW ratio reflects how aggregate facility retention from the current ART cohort size is measured and compared to the number of new initiations for the period under consideration. TXCURR is defined as the current ART cohort size of all active patients. A ratio less than one indicates a loss of patients; equal to one indicates no loss of patients; and a net new ratio greater than one indicates that existing patients categorized as lost to follow-up were brought back into care.

Monthly data captured through the RTC KC was classified by facility type (Siyenza or non-Siyenza). Summary statistics were calculated for each measure and facility classification. We then compared each class's post-intervention indicator measures to a historical baseline (pre-Siyenza) to calculate percent change.

PEPFAR Indicators were calculated for patients initiated on ART (TX_NEW), net retention (TX_NET_NEW) and the net retention in care or TX NET NEW ratio as described in Eq. 1.

Data were cleaned and outliers were removed in SPSS v 26 (IBM Corp. 2013, Armonk, NY). A retention in care ratio (TX_NET_NEW ratio) of 18.0 was removed from the values for Siyenza and a retention of care ratio of 44.0 was removed from the non-Siyenza retention in care values. Shapiro Wilk tests were performed to establish whether indicators were normally distributed. The latter indicated whether data required further transformation. The analysis indicated that non-parametric analysis was possible for initiation on ART and the net increase or decrease of patients. It was possible to transform the net retention ratio in Eq. 1 to a normal curve using a Box-Cox transformation in SPSS, thereby allowing for prediction of the TX_NET_NEW ratio for both periods using a linear regression and a t-test to determine whether there are significant differences between the means. The application of regression weights was used to correct for selection bias. The Common Language effect size for the TX_NET_NEW Ratio was calculated as described in (8):

$\mathrm{Z}=|\mathrm{X} 1-\mathrm{X} 2| /\{\{\mathrm{SD} 2 / 1\}+\{\mathrm{SD} 2 / 2\}\}$

\section{Results}

For these analyses, cohort outcome results are categorized as pre-intervention (Dec 2018 - Feb 2019) and intervention (March 2019 - May 2019 ) results for both Siyenza and non-Siyenza selected sites.

A limitation of the analysis is that the results after the intervention period cannot be compared to the period before the intervention since the Department of Health continued with the intervention indefinitely. 
Table 1

Summary Results for the comparison between the pre-intervention and intervention period in Siyenza and non-Siyenza facilities

\begin{tabular}{|c|c|c|c|c|c|c|}
\hline \multirow{3}{*}{ Measure } & \multirow{3}{*}{$\begin{array}{l}\text { Pre-Intervention } \\
\text { Dec 2018-Feb } 2019\end{array}$} & \multicolumn{2}{|c|}{ Non-Siyenza facilities $(n=29)$} & Siyenza $\mathrm{f}$ & Siyenza facilities (n-181) & \multirow[b]{2}{*}{$\%$ Change } \\
\hline & & Intervention & $\%$ Change & Pre-Intervention & Intervention & \\
\hline & & March-May 2019 & & Dec 2018-Feb 2019 & March-May 2019 & \\
\hline \multicolumn{7}{|l|}{ Mean value per facility } \\
\hline Monthly TX_NEW & 54 & 62 & $16 \%$ & 14 & 16 & $13 \%$ \\
\hline TX_CURR & 3057 & 3272 & $7 \%$ & 1104 & 1141 & $3 \%$ \\
\hline TX_NET_NEW & 81 & 127 & $57 \%$ & 31 & 15 & $-52 \%$ \\
\hline \multicolumn{7}{|l|}{ Total value for the period } \\
\hline Total TX NEW & 4675 & 5427 & $16 \%$ & 7415 & 8347 & $13 \%$ \\
\hline Total endpoint TX_CURR & 88645 & 94874 & $7 \%$ & 198766 & 205317 & $3 \%$ \\
\hline Total NET_NEW & 2346 & 6229 & & 5596 & 6551 & \\
\hline
\end{tabular}

A net increase or decrease of patients (TX_NET_NEW) before the intervention (Dec 2018 - Feb 2019) was compared to the time-period when the intervention occurred (March 2019 to May 2019) in both Siyenza sites and non-Siyenza sites.

As described by Table 1, ART initiations increased for both Siyenza-targeted facilities and non-targeted facilities (16\% and $13 \%$ respectively). There was an average monthly ART initiation of 62 patients per facility during the intervention period in Siyenza-targeted facilities compared to a pre-intervention monthly facility average of 54 ART initiations. However, significant gains in ART retention were observed in Siyenza-targeted facilities calculated as the net new retention in care ratio. Siyenza-targeted facilities recorded a total cohort growth of $7 \%(n=6,229)$ over the three months of observation, compared to $3 \%$ in nontargeted facilities. Consequently, the retention improved dramatically in Siyenza-targeted facilities: the net retention in care ratio increased from 0.68 at baseline to 1.15 after three months of program intervention. The non-targeted facilities, representing smaller per facility cohorts, observed a decrease in retention; prior to the Siyenza intervention, non-targeted sites were retaining as many patients as were initiated (net new retention ratio of 1.02 ). Retention decreases to a net retention in care ratio value of 0.78 during the Siyenza intervention. The results indicate that caution should be used not to let up on nonselected facilities whilst focusing on intervention facilities.

Equation 1 for the non-Siyenza and Siyenza period or the net new retention in care ratio (TX_NET_NEW ratio) indicates the net new number of patients (TX_NET_NEW) for the number of patients newly initiated on ART (TX_NEW) for the period considered. This measure does not provide information on the size (ab-solute number) of the people no longer counted or whether the size of loss is within acceptable limits of non-retention.

Figure 2 describes the immediate effect of the Siyenza interventions for both non-Siyenza and Siyenza facilities. The Siyenza facilities maintained positive cohort growth, while non-Siyenza facilities continued to lose patients.

Correlation tests were conducted to examine the relationship between retention and selected Siyenza facilities before and during the period when the Siyenza intervention was conducted. As per Cohen's guidelines in (9), a small correlation is $r=.10$ to .29 , medium $r=.30$ to .49 and large $r=.50$ to 1.0 . A small positive correlation of 0.153 was recorded during the Siyenza period between Siyenza facilities and retention measured with Eq. 1. Similarly, a small positive correlation of 0.115 was noted between non-Siyenza facilities and retention for the non-Siyenza period.

Shapiro-Wilk tests for normality indicated that the data for the TX_NET_NEW Ratio were not normally distributed for 180 non-intervention sites in March to May $2019(.354, p=0.000)$ in the non-intervention period and in the intervention period it was $(0.068, p=0.000)$. The 29 Intervention sites were normally distributed $(0.991, p=0.997)$ during the pre-intervention and intervention period $(0.938, p=0.091)$.

The data for the TX_NET_NEW Ratio in the period before and during Siyenza interventions were not normally distributed therefore it required a Box-Cox transformation to enable a regression and independent sample t-tests to be performed.

A regression indicated that the net retention scores (TX_NET_NEW Ratio) or Eq. 1: for the period during the intervention explained a significant amount of the variance $(15 \%)$ in the value of retention from baseline for three months $(F=4.948, p<0.027, R 2=0.23, R 2$ adjusted $=-0.19)$, with $p<0.05$.

Figure 3 indicates that the net retention in care ratio for non-Siyenza facilities was 0.29 in comparison to a ratio of 1.66 in Siyenza facilities before interventions were implemented. Further losses in retention were predicted for non-Siyenza facilities to 0.23 during March- to May 2019 , whilst a gain was expected in the net retention ratio in Siyenza facilities of 1.21 , meaning that patients were not only retained in care, but were brought back to care through tracing efforts.

Results of the Independent-samples t-test

An independent samples t-test was run to determine whether there are significant differences between the net retention in in care (TX_NET_NEW Ratio) during the intervention in Siyenza and non-Siyenza facilities after outliers have been removed as described in the methodology. The independent-samples t-test indicated that the net retention in care ratio (TX_NET NEW Ratio scores) were significantly higher for Siyenza facilities $(M=0.379, S D=0.808)$ compared to 
non-Siyenza facilities $(M=0.061, S D=1.016), t(208)=2.224, p<0.027, d=0.445$ during the Siyenza period. Homogeneity of variances was not violated, as assessed using a Levine's test $(p=0.776)$. The results of the independent-samples t-test indicated that there was indeed a significant difference between the means of the two different groups with a Common Language $(\mathrm{CL})$ effect size of 0.623 . Consequently, this means that a measurement from the intervention group have a $62,3 \%$ probability of having a higher observed mean net retention in care than the non-intervention group during the Siyenza period.

\section{Discussion}

A suite of data and clinical interventions was identified and implemented as part of the Siyenza program in Ehlanzeni, South Africa and daily data review meetings resulting in improved ART initiation and retention in care compared to the period immediately preceding Siyenza. Interventions included improved facility data management (file audits, workflow improvements and same day data entry, and standardized filing system) including the application of clinical best practices (increased multi-month scripting and dispensing, enhanced track, and trace for lost to follow-up patients, extended clinic hours, and appointment reminders and follow-up for early missed appointments). The program has since established a tracking system to better link these interventions with facility outcomes.

The availability and actioning of almost real-time data are critical to demonstrating the impact of the rapid program improvements described above and targeting those interventions to the right facilities. Program data in large HIV care and treatment programs are currently relegated to providing retrospective program evaluation at an aggregated level. However, to meet increasingly ambitious program performance targets, care, and treatment partners with authorized users must be able to access, process and act on daily data from multiple integrated data sources, preferably at patient level to avoid double data entry.

As the Siyenza intervention began, program managers identified several data gaps related to human re-sources, timely procurement, supply chain management, data flow and quality assurance issues. These data are often captured in other systems (e.g., Workload Indicators for Staffing Needs, Synch, Rx Solution, etc.) supporting the need for further system integration. The data should be at the lowest level possible, bearing in mind program efficiency, feasibility, quality, and cost (10). Any mHealth or e-Health programs should consider evaluation from the outset, including collection of baseline data prior to implementation.

Leadership promoting daily data review of progress against targets for key indicators at priority facilities worked to reinforce a culture of data utilization for Right to Care as has been demonstrated to be effective elsewhere (11). Multi-disciplinary data review meetings should allow rapid review of the data and decision-making, including tapping into the tacit knowledge of a diverse team. Data managers and program managers are then cooperatively able to interrogate the trends and interpret real-time data (aware of inconsistencies and quality implications of this type of data) to adjust resources and propose tailored interventions. The rapidity of the data and review allow for quick evaluation of the interventions that are being implemented at struggling facilities.

All care and treatment partners are expected to submit similar data to funders; however, during the intervention the process was previously overly manual and required multiple points of data capture, categorization, and entry. This time-consuming process limited any further analysis that could have been performed by our staff to focus on program improvement. Automating certain steps in the reporting and analytic process with Right to Care's Knowledge Centre will allow for more time to interpret and act on the data, which is essential when using daily data. Automated reporting templates ensure standardized data from every facility with reports mimicking the required reporting forms and simplifying transcription; and automated visualizations provide up-to-date data with every form submission.

If the granularity and frequency of data collection is overly burdensome then reporting compliance and data quality can suffer. A near-real time system can expect to have some data quality trade-offs: when data is reported more frequently, an invalid data point is diluted and mitigated as an outlier. Focusing on data recency (the time elapsed between event and reporting) allows for very timely correlation between intervention and program outcome.

Improving the efficiency of the data management and analysis provide time to interpret and act on the data. The latter a driving factor to improved program performance and sustaining daily review meetings. Data review meetings allow program managers to select evidence-based interventions based on the facility need. The interventions' scopes vary from clinical technical assistance, increased human resources, file management and data quality, and supply chain efficiencies. The recency of the data and frequency of review also enable rapid evaluation of the chosen interventions.

The process that we developed and implemented for our programs in South Africa still relies on an inter-mediate data transcription process - from clinical registers, paper files, and electronic medical records into the Knowledge Centre data collection forms. Right to Care has demonstrated the ability to integrate testing, clinical, and laboratory databases leveraging the investments made to capture clinical data in electronic systems. All care and treatment partners and the Department of Health could benefit by further providing governance on how electronic clinical data can be accessed, integrated, and used. Standards for application programming interfaces and interoperability of systems (12)(13)(14) could further create a data eco-system that is broader than government facilities and truly tracks patients across systems to ensure their outcomes are achieved as programs meet performance targets more efficiently and effectively.

Democratizing the ethical use and integration of electronic disaggregated patient-level clinical data can potentially propel HIV care and treatment into new frontiers with segmentation to truly understand patient needs as well as predictive modelling. We can then start to strengthen the governmental support at the district and provincial level through regular access to analytics covering the care and treatment cascade. We can also begin to tailor patient treatment protocols (inclusive of psychosocial support) at the point of testing based on their potential for poor outcomes like being lost from care. Providing advanced analytics as a service to government and supporting partners puts greater focus on effective and precise interventions. Data managers can then focus on ensuring that quality data is captured on time for every patient into the primary data source rather than a myriad of alternative data collection systems. The integration of systems will result in less duplication and better efficiency after innovations and legacy risks have been assessed.

Page $5 / 9$ 
The positive results from the study indicate that accessibility and utilization of near to real-time data within HIV programs allow for rapid responses to program performance, needs and improved patient outcomes. Daily situation rooms and reviews, facilitates precision programming, and contributes to the behavior change necessary to institute routine data use for decision making and evaluations within programs. Continued investment in real-time data sources and automated analytic and visualization systems are essential to ensuring programs meet their aggressive goals and the individual needs of patients to stay in care.

Integration or a (15) Health Information Exchange (HIE) has the potential to close many of the gaps that lead to sub-optimal care for people with HIV by, for example, improving the referral and tracking of patients among services, identifying patients who have fallen out of care in a health system, and allowing providers to coordinate services to ensure that every individual receives the optimal services needed.

Information provision and communication of non-conflicting messages, facilitated through workshops to inform or update on the synthesized evidence and subsequent recommendations, result in successful implementation (11) as demonstrated in the Siyenza results.

\section{Conclusions}

The accessibility and utilization of near to real-time patient-level data within HIV programs allow for rapid responses to program performance, including an improved understanding of patient needs and improved patient outcomes. Daily situation rooms and reviews, facilitates precision programming, and contributes to the behavior change necessary to institute routine data use for decision making and evaluations within programs. Accessibility of information linked to the ability to establish a real-time HIE would assist even further in improving HIV interventions and patient outcomes. Text and results for this section, as per the individual journal's instructions for authors.

\section{Abbreviations}

\begin{tabular}{|ll|}
\hline Abbreviation & Explanation \\
\hline ART & Antiretrovirals \\
\hline Cohort & A group similar in characteristics that is part of a study observed over time \\
\hline HIE & $\begin{array}{l}\text { Health Information Exchange where health care information is mobilized electronically across organizations within a region, community, or } \\
\text { hospital system }\end{array}$ \\
\hline M & Mean \\
\hline SD & Standard Deviation \\
\hline TX NEW & Number of adults and children newly enrolled on antiretroviral therapy (ART) - usually quarterly \\
\hline TX_CURR & Number of adults and children currently receiving antiretroviral therapy (ART) \\
\hline
\end{tabular}

\section{Declarations}

Acknowledgements

The authors of the document (not in order of importance) acknowledge the inputs of the Monitoring and Evaluation Officers in Right to Care, the counsellors, the data capturers, and the Strategic Information Office of Right to Care who contributed as this is a product of Right to Care. Thanks also for all the effort of our district colleagues and the facility managers in Thabo Mofutsanyane and Ehlanzeni. The operational research evaluation was conducted by Right to Care that are receiving USAID funding for the implementation of the APACE project. Special thanks for the input of Dr. Heena Brahmbhatt from USAID. This document would not have been possible without the input and support of the Department of Health in Mpumalanga, and everyone involved.

Funding

USAID GRANT NUMBER - $72067418 C A 00029$ for APACE Grant. No funding for the article.

Availability of data and materials

The authors can be contacted for availability of the data and materials.

Ethics approval and consent to participate

Not applicable. Routine monitoring and evaluation data.

Competing interests

The authors declare that they have no competing interests.

Consent for publication

Consent was provided by Provincial Health Department in Mpumalanga 
P.T.P conceptualized, coordinated, and drafted the manuscript. M.A.P contributed to writing, analyzed, interpreted the data, and wrote parts of the report. M.R interpreted and provided input into the manuscript, H.B revised the manuscript, C.O. coordinated and provided input into the draft. T.M. coordinated electronic systems, A.C reviewed the analysis, P. Majuba, I.S, D.M, B.M, I.M and P. Mogane revied the manuscript for intellectual content. All authors reviewed and approved the manuscript.

Author's information

${ }^{1}$ Strategic Information, Right to Care, Centurion, South Africa. ${ }^{2}$ Health Office, USAID, Pretoria, South Africa. ${ }^{3}$ Ehlanzeni District Office, Department of Health, Mbombela, South Africa. ${ }^{4}$ Provincial Office, Mpumalanga Department of Health, Mbombela, South Africa

\section{References}

1. PEPFAR (US President's Emergency Plan for AIDS Relief). Monitoring, Evaluation, and Reporting Indicator Reference Guide. Washington; 2014.

2. Marsh K, Eaton JW, Mahy M, Sabin K, Autenrieth CS, Wanyeki I, et al. Global, regional, and country-level 90-90-90 estimates for 2018: Assessing progress towards the 2020 target. AIDS. 2019 Dec;33(Suppl 3):S213-26.

3. Havlir D, Lockman S, Ayles H, Larmarange J, Chamie G, Gaolathe T, et al. What do the Universal Test and Treat trials tell us about the path to HIV epidemic control? J Int AIDS Soc. 2020 Feb;23(2).

4. Bhattarai S, Shah RK. Leveraging real-time data to drive quality improvement in a pediatric health system. Pediatr Investig. 2018;2(3):184-7.

5. Virga PH, Jin B, Thomas J, Virodov S. Electronic health information technology as a tool for improving quality of care and health outcomes for HIV/AIDS patients. Int J Med Inform. 2012 Oct;81(10):e39-45.

6. Nash D, Elul B, Rabkin M, Tun M, Saito S, Becker M, et al. Strategies for More Effective Monitoring and Evaluation Systems in HIV Programmatic Scale-Up in Resource-Limited Settings: Implications for Health Systems Strengthening. JAIDS J Acquired Immune Deficiency Syndrome. 2009 Nov;52(SUPPL. 1):S58-62.

7. Statistics South Africa. South African Statistics Quality Assessment Framework (SASQAF) [Inter-net]. 2010. 12 p. Available from: http://www.statssa.gov.za/standardisation/SASQAF_Edition_2.pdf

8. Lakens D. Calculating and reporting effect sizes to facilitate cumulative science: A practical primer for t-tests and ANOVAs. Front Psychol. 2013;4(NOV):1-12.

9. Pallant J. SPSS Survival Manual 2nd Edition [Internet]. 2005. Available from:

10. Peter J, Benjamin P, LeFevre AE, Barron P, Pillay Y. Taking digital health innovation to scale in South Africa: Ten lessons from MomConnect. BMJ Glob Heal. 2018;3:2-5.

11. Ravhengani NM, Mtshali NG. The Views of Health Workforce Managers on the Implementation of Workload Indicators of Staffing Need (WISN) Method in Primary Healthcare Settings in South Africa. Int J Stud Nurs. 2017;3(1):132.

12. Ngwenya M. Health Systems Data Interoperability and Implementation [Internet]. University of South Africa; 2018. Available from: https://www.academia.edu/38922036/The_Integration_of_Technology_into_English_Language_Teaching_The_Underlying_Significance_of_LMS_in_ESL_T https://doi.org/10.1155/2016/315980

13. Systems GI. Minimum Interoperability Standards (MIOS) for Government Information Systems. Technology. 2011;(November).

14. NDOH. South African National Health Normative Standards Framework for Interoperability in eHealth Government Gazette Version - March 2014. 2014; (March):1-57. Available from: http://www.health.gov.za/docs/ehealth/ehealth-Standards-Report-V2-March 2014.pdf

15. Shade SB, Steward WT, Koester KA, Chakravarty D, Myers JJ. Health information technology interventions enhance care completion, engagement in HIV care and treatment, and viral suppression among HIV-infected patients in publicly funded settings. J Am Med Informatics Assoc. 2014;22(e1):e104-11.

\section{Figures}




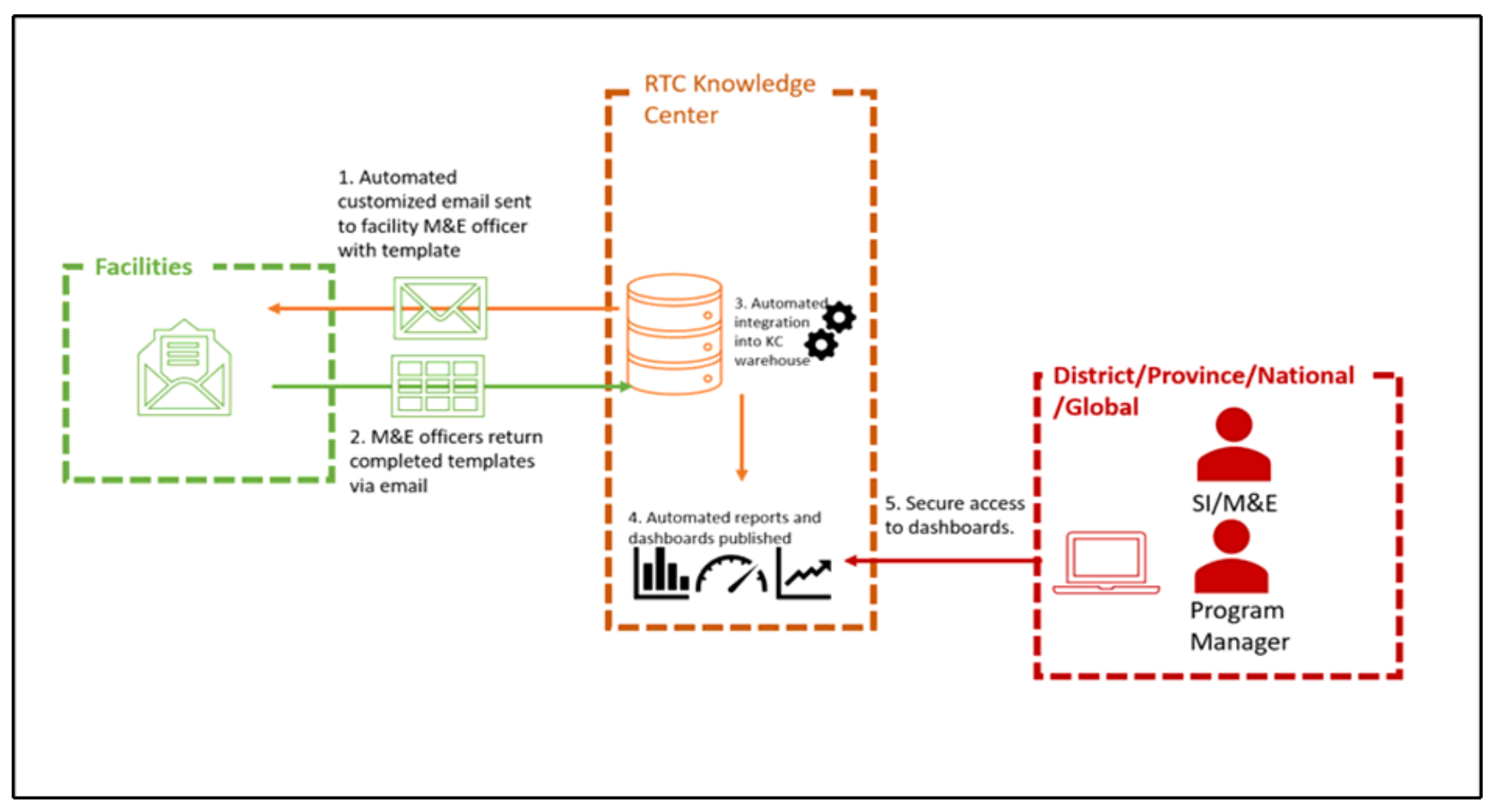

Figure 1

Process flow diagram for the daily Automated Data Capture templates

\subsection{0}
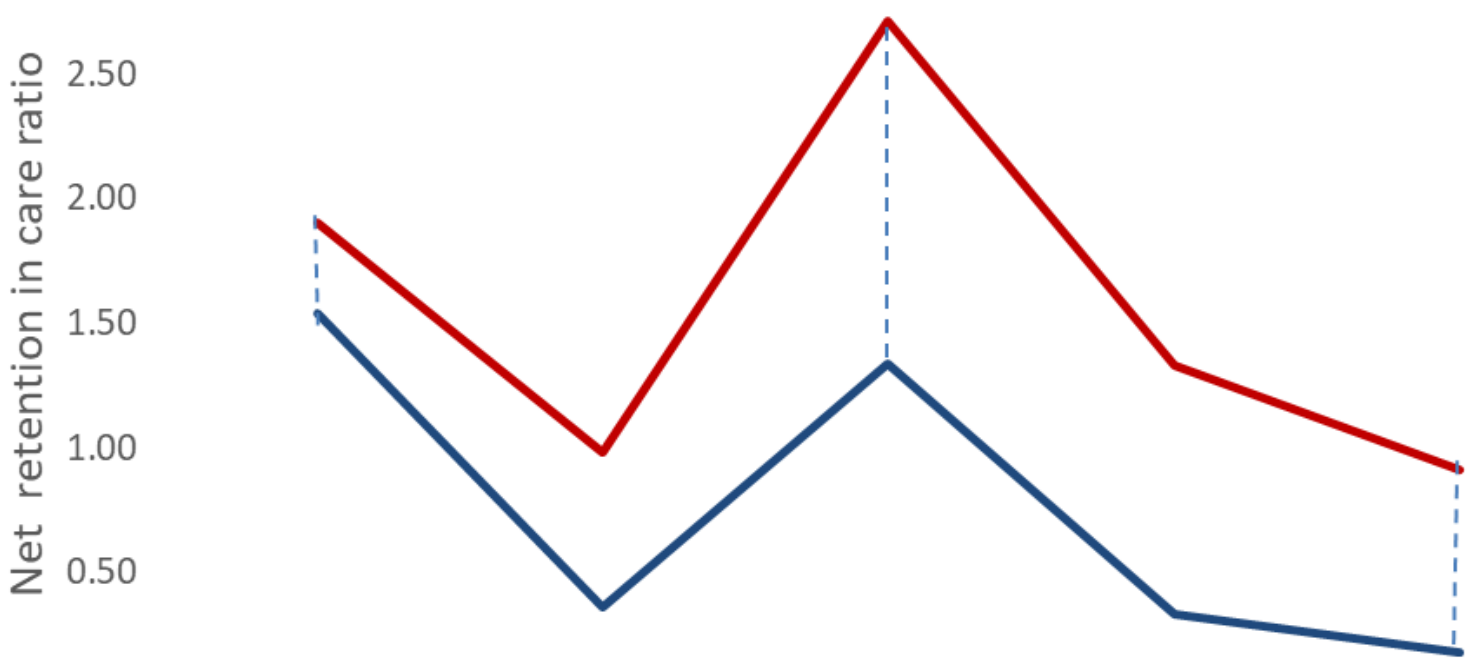

0.00

Jan

Feb

March

Apr

May

Monthly period

-Non-Siyenza facilities —-Siyenza facilities $: \begin{aligned} & \text { Intervention } \\ & \text { period }\end{aligned}$

Figure 2

Comparison between monthly retention in care (NET NEW) ratios calculated as described (monthly) 


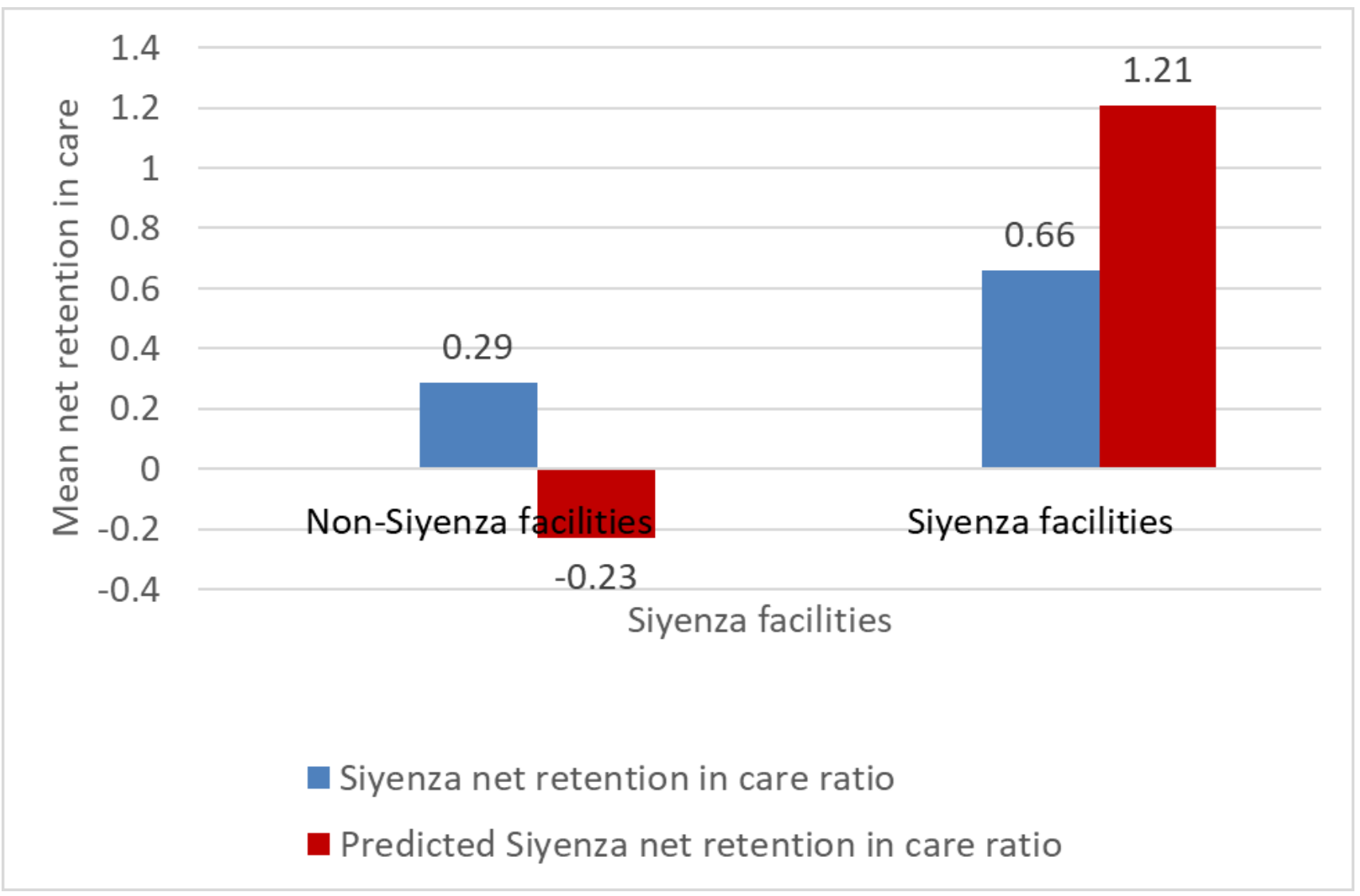

Figure 3

Results of a regression to predict the net retention in care (TX_NET_NEW Ratio) for a period after the intervention period. 